plausible causal inference to guide action, while accepting and adapting to the reality of the public health landscape rather than wishing it were otherwise. The traditional art of dry stone walling can serve as a metaphor for the more 'holistic sense-making' we propose.

\section{P39 EVALUATIONS OF PUBLIC HEALTH INTERVENTIONS USING NATURAL EXPERIMENT EVALUATION DESIGNS AND THE 'TARGET TRIAL' FRAMEWORK}

\footnotetext{
1,2,3 Frank de Vocht* ${ }^{*}{ }^{4}$ Srinivasa Vittal Katikireddi, ${ }^{1,2}$ Cheryl McQuire, ${ }^{1,5}$ Kate Tilling, ${ }^{1}$ Matthew Hickman, ${ }^{4}$ Peter Craig. ${ }^{1}$ Population Health Sciences, University of Bristol, Bristol, UK; ${ }^{2}$ MRC/CSO Social and Public Health Sciences Unit, University of Glasgow, Glasgow, UK; ${ }^{3}$ MRC IEU, University of Bristol, Bristol, UK
}

\subsection{6/jech-2021-SSMabstracts. 127}

Background Natural or quasi experiments are appealing because they enable the evaluation of events or interventions that are difficult or impossible to manipulate experimentally, which is especially relevant for public health research in which the evaluation of policy and health system reforms is an important focus. There remains ambiguity about their definition and how they differ from randomized controlled experiments and from other observational designs. We conceptualise natural experiments in the context of public health evaluations and align the study design to the Target Trial Framework.

Methods A literature search was conducted, and key methodological papers were used to develop this work. Peer-reviewed papers were supplemented by grey literature.

Results Natural experiment studies (NES) combine features of experiments and non-experiments. They differ from planned experiments, such as randomized controlled trials, in that the assignment of exposure is not controlled by researchers. They differ from other observational designs in that they evaluate the impact of events or processes that resulting from changes in exposure. As a result they are, in theory, less susceptible to bias than other observational study designs. Importantly, causal inference relies heavily on the assumption of 'as-if randomisation' of exposure allocation. The target trial framework provides a systematic basis for evaluating this assumption and the other design elements that underpin the causal claims that can be made from NES.

Conclusion Although there will always remain some ambiguity about the strength of causal claims from natural experiment evaluations, there are clear benefits to harnessing these rather than relying purely on observational studies. This includes the fact that NES can be based on routinely available data and that timely evidence of real-world relevance can be generated. Aligning NES to the Target Trial framework will guard against conceptual stretching of these evaluations and ensure that the causal claims about whether public health interventions 'work' can inform public health action within a 'practice-based evidence' framework.

\section{P40 IS THERE AN EVIDENCE BASE ON REDUCING LIFESTYLE RISK BEHAVIOURS IN DISADVANTAGED GROUPS? A SCOPING REVIEW OF SYSTEMATIC REVIEWS}

${ }^{1}$ Mark Rodgers* ${ }^{1}$ Emily South, ${ }^{1}$ Kath Wright, ${ }^{2}$ Margaret Whitehead, ${ }^{1}$ Amanda Sowden. ${ }^{1}$ Centre for Reviews and Dissemination, University of York, York, UK; ${ }^{2}$ Department of Public Health and Policy, University of Liverpool, Liverpool, UK

10.1136/jech-2021-SSMabstracts. 128
Background Lifestyle risk behaviours are associated with an increased risk of non-communicable disease and mortality. There are socio-economic inequalities in these behaviours, with some behaviours being more prevalent in particular groups, such as prisoners, homeless people and Gyspies, Travellers and Roma. The aim of this scoping review was to identify and bring together existing evidence from systematic reviews on reducing risk behaviours in disadvantaged groups and highlight where there is insufficient evidence to inform policy.

Methods MEDLINE and Embase were searched up to October 2020 for English language reviews, with supplementary searching in Epistemonikos and Health Systems Evidence. Systematic reviews reporting behavioural outcomes of interventions targeting smoking, excessive alcohol use, unhealthy diet or physical inactivity in low income or socio-economic status (SES), unemployed people, homeless people, care leavers, prisoners, refugees or asylum seekers, Travellers, Gypsies or Roma, people with learning disabilities or deprived areas or communities were eligible. Reviews of population-level policies reporting differential effects for disadvantaged groups and qualitative reviews exploring barriers or facilitators to behaviour change were also included. The literature was mapped based on the group and behaviour targeted.

Results In total 9,336 records were screened, 262 full texts retrieved and 92 systematic reviews included. The majority of reviews included studies of people with low income or SES $(n=68)$, with diet and low income the most frequently addressed combination. There were fourteen reviews on prisoners, 12 on deprived areas, ten on homeless people and nine on people with learning disabilities. Only three reviews included unemployed people and two included refugees or asylum seekers (both focusing on barriers and facilitators to healthy eating). No reviews were identified on care leavers or Gypsies, Travellers and Roma. In total there were 11 reviews targeting alcohol use. Sixteen qualitative reviews explored participants' perceptions of barriers and facilitators to changing their behaviour.

Conclusion A large number of systematic reviews were identified but we found some evidence gaps where new syntheses or primary studies may be needed to guide policy, for example on care leavers, Gypsies, Travellers and Roma and refugees and asylum seekers. Other useful contributions might include an overview bringing together different interventions in lowincome populations and an overview of the perceptions of disadvantaged groups about behaviour change, allowing common barriers to be identified as well as factors that are unique to specific groups.

\section{P41 A SYSTEMATIC REVIEW OF INEQUALITIES IN THE UPTAKE OF, ADHERENCE TO, AND EFFECTIVENESS OF BEHAVIOURAL WEIGHT MANAGEMENT INTERVENTIONS IN ADULTS}

${ }^{1}$ Jack Birch*, ${ }^{1}$ Rebecca Jones, ${ }^{1}$ Julia Mueller, ${ }^{2}$ Matthew McDonald, ${ }^{1}$ Rebecca Richards, ${ }^{3}$ Michael Kelly, ${ }^{1,3}$ Simon Griffin, ${ }^{1}$ Amy Ahern. ${ }^{1}$ MRC Epidemiology Unit, University of Cambridge, Cambridge, UK; ${ }^{2}$ Curtin School of Population Health, Curtin University, Perth, Australia; ${ }^{3}$ Primary Care Unit, University of Cambridge, Cambridge, UK

\subsection{6/jech-2021-SSMabstracts. 129}

Background Health inequalities are a public health priority. The extent to which behavioural weight management interventions impact health inequalities is uncertain, as is the extent 